Research Paper

\title{
Zusanli (ST36) Acupoint Injection with Neostigmine for Paralytic Postoperative lleus following Radical Gastrectomy for Gastric Cancer: a Randomized Clinical Trial
}

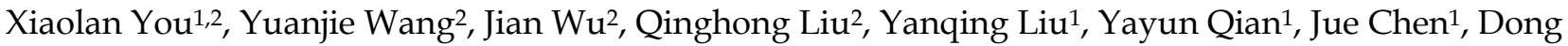 \\ Tang 3 , Daorong Wang ${ }^{3 凶}$ \\ 1. Department of Integrated Traditional Chinese and Western Medicine, Medical College of Yangzhou University. Yangzhou, Jiangsu Province, China \\ 2. Department of Gastrointestinal Surgery, Taizhou people's Hospital, Taizhou, Jiangsu Province, China \\ 3. Department of Gastrointestinal Surgery, Clinical Medical College of Yangzhou University, Subei People's Hospital of Jiangsu Province, Yangzhou Jiangsu \\ Province, China
}

$\square$ Corresponding author: Daorong Wang, Department of Gastrointestinal Surgery, Clinical Medical College of Yangzhou University, Subei People's Hospital of Jiangsu Province, Yangzhou, Jiangsu Province 225000, China. e-mail: wdaorong666@sina.com

(c) Ivyspring International Publisher. This is an open access article distributed under the terms of the Creative Commons Attribution (CC BY-NC) license (https://creativecommons.org/licenses/by-nc/4.0/). See http://ivyspring.com/terms for full terms and conditions.

Received: 2018.01.05; Accepted: 2018.03.16; Published: 2018.06.05

\begin{abstract}
Background: The Zusanli (ST36) acupoint has been associated with treatment of various gastrointestinal conditions. There have been no studies of acupuncture therapy for paralytic postoperative ileus (PPOI).

Materials and methods: Patients with PPOI following gastrectomy for gastric cancer were randomized to receive ST36 acupoint injection with neostigmine, gluteal intramuscular injection with $1.0 \mathrm{mg}$ neostigmine, ST36 acupuncture alone, or standard therapy. The main outcome was the effectiveness rate for recovery of peristalsis. Secondary outcomes were time to bowel sound recovery, time to first flatus, and time to first defecation. Tertiary outcomes were drug-related adverse events, including abdominal pain, diarrhea, nausea, vomiting, tearing, delirium, seizure, and anxiety.
\end{abstract}

Results: ST36 acupoint injection with neostigmine and gluteal intramuscular injection of neostigmine gave a higher rate of peristalsis recovery, and the ST36 acupoint injection group showed significantly higher total effectiveness rate than that of the intramuscular injection group. These interventions gave significantly shorter times to bowel sound recovery, shorter times to first flatus and first defecation compared with ST36 acupuncture and standard post-operative therapy $(P$ $<0.01$ ). ST36 acupoint injection group gave shorter time to bowel sound recovery, shorter time to first flatus and first defecation than those of the intramuscular injection group $(P<0.01)$. Drug-related adverse events in the intramuscular injection group were more serious than in the ST36 acupoint injection group $(P<0.05)$.

Conclusion: ST36 acupoint injection with neostigmine is safe and effective for treatment of PPOI.

Key words: gastric cancer, paralytic postoperative ileus, ST36 acupucture, ST36 acupoint injection

\section{Introduction}

Postoperative ileus (POI) is a common condition following gastrectomy [1,2]. It is characterized by abdominal discomfort, pain, nausea, vomiting, and distension [2]. POI is generally defined as temporary
(< 3 day) impairment in gastrointestinal motility following surgery $[3,4]$. If motility does not recover within the expected interval, it is defined as paralytic postoperative ileus (PPOI) [5]. PPOI is further 
characterized by a range of further complications $[6,7]$. Patients cannot be discharged from the hospital until the ileus has resolved, thus extending hospitalization, and increasing costs [2].

PPOI following radical gastrectomy for gastric cancer gives rise to bloating, atelectasis, pneumonia, and the elevated risk of other postoperative complications [8-10]. Therefore, prompt restoration of bowel function is especially important following radical gastrectomy for gastric cancer.

Currently, treatment for PPOI in China is supportive. Standard care includes fasting, nasogastric suction, intravenous hydration, parenteral nutrition, and gradual ambulation with simple exercises. Pharmacological interventions include neostigmine, laxatives, erythromycin, naloxone, beta blockers, metoclopramide and Chinese herbal medicine [11,12]. However, these interventions have limited clinical efficacy and safety $[13,14]$.

Acupuncture has been used widely in clinical practice for at least 3000 years [15], and is used in at least 78 countries [16]. The commonly-used acupoint to treat gastrointestinal diseases is Zusanli (ST36) [17]. ST36 is located on the stomach meridian. It is thought to act on the spleen and stomach, and to harmonizes qi and blood. Acupuncture at Zusanli (ST-36) may improve upper and lower abdominal symptoms and restore impaired gastric slow waves, which are induced by rectal distension, via the vagal pathway [18]. Its traditional therapeutic properties are ideally suited for treatment of PPOI [19-21]. Acupoint injection therapy originated from intramuscular injection in Western medicine and was gradually integrated into traditional Chinese medicine [22]. It is an acupoint stimulating technique in which a liquid agent is injected to prevent or treat disease. Agents injected in acupoints, through the meridians, are considered to exert synergistic effects with acupoint stimulation, and are thought to give more sustained effects than traditional acupuncture needling, or simple intramuscular injection [23].

ST36 acupuncture or ST36 acupoint injections with various agents have been widely used for prevention of POI in China for many years. However, the technique has not been evaluated for efficacy following radical gastrectomy for gastric cancer (GC). Therefore, we conducted this randomized clinical trial comparing ST36 acupoint injection with neostigmine, intramuscular injection with neostigmine, ST36 acupuncture, and standard therapy for PPOI. We measured the effectiveness rate, time to bowel sounds recovery, time to first flatus or first defecation, and the drug- related adverse events were followed following therapy.

\section{Patients and Methods}

Participants were recruited between September 2014 and March 2017 from the Department of Gastrointestinal Surgery of our institute. Patients were asked to participate if they had been diagnosed with primary GC and had been scheduled for resection, whether laparoscopic assisted or open. Inclusion criteria were as follows: (1) radical gastrectomy and D2 lymphadenectomy; (2) absence of flatus or defecation from postoperation day 3 (POD3) to POD 6; (3) quiet abdomen with few bowel sounds on auscultation; and (4) symptomatic bloating and distention. Exclusion criteria included: (1) age younger than 18 years; (2) history of major abdominal or pelvic surgery; (3) pregnancy or lactation; and (4) signs of peritoneal irritation (abdominal tenderness and tension, or rebound tenderness); (5) allergy to acupuncture needles; (6) history of chronic disease including coronary artery disease, epilepsy, ventricular tachycardia, asthma, hyperthyroidism, and renal insufficiency; and (7) psychological or social conditions that might interfere with their participation. The protocol of this trial was designed in accordance with the Declaration of Helsinki and was approved by the Clinical Research Ethics Committee of our institute (TZRY-CR-14-0606). All patients were provided details regarding the assessment procedure, and all gave informed written consent.

\section{Randomization and Blinding}

Following surgery, we used TenAlea software to randomize patients with equal probability into various group if the patient met all eligibility criteria. Participants were randomly on a 1:1:1:1 basis to the four arms: ST 36 acupuncture, receiving acupuncture treatment once a day; ST36 acupoint injection with neostigmine (a total of $1.0 \mathrm{mg}, 0.5 \mathrm{mg}$ per side); intramuscular injection with $1.0 \mathrm{mg}$ neostigmine; or standard therapy.

Given the obviously different characteristics of ST36 acupuncture, ST36 acupoint injection, and intramuscular injection, no blinding was applied to participants or physicians. Nevertheless, the nursing staff and statisticians were blinded to treatment allocation throughout data collection and analysis.

\section{Procedures}

All radical gastrectomies were performed by senior surgeons with over 20 years of experience. All patients underwent total intravenous anesthesia. All refused combined spinal-epidural plus epidural anesthesia. All received identical standardized protocols for perioperative management and postoperative care. $250 \mathrm{ml}$ tepid normal saline was administered via 
naso-jejunal tube on the first postoperative morning. $500 \mathrm{ml}$ tepid enteral nutrient solution was administered via NJ tube starting on the second postoperative morning, and once daily thereafter.

Three nurses and two surgeons from Department of Gastrointestinal Surgery of our institute spent 2 months training for this study. A senior acupuncturist with over 30 years' acupuncture experience trained the research nurses and surgeons to point location. Surgeons trained the research nurses in auscultation and observation of abdominal signs. An experienced statistician was involved in all stages of study development and analysis.

\section{ST36 Acupuncture}

Patients in the ST 36 acupuncture group received acupuncture treatment once a day. Treatments were performed in the patient's bed by a senior acupuncturist with over 30 years of experience. ST36 is located on the lateral surface of the leg, 3 cun distal to the lower border of the patella, 1 finger-breadth lateral to the anterior crest of the tibia, between the tibialis anterior muscle and the tendon of the extensor digitorum longus (Figure 1). (The breadth of patient's middle finger was the proportional unit of measure "the cun", defined as the distance between the two medial ends of the creases of the interphalangeal joints when the patient's middle finger is flexed).

Disposable, single-use, sterilized acupuncture needles were used (Huatuo, Suzhou, China) conforming to the requirements of the Australia TGA, United States FDA, Germany TUG, International

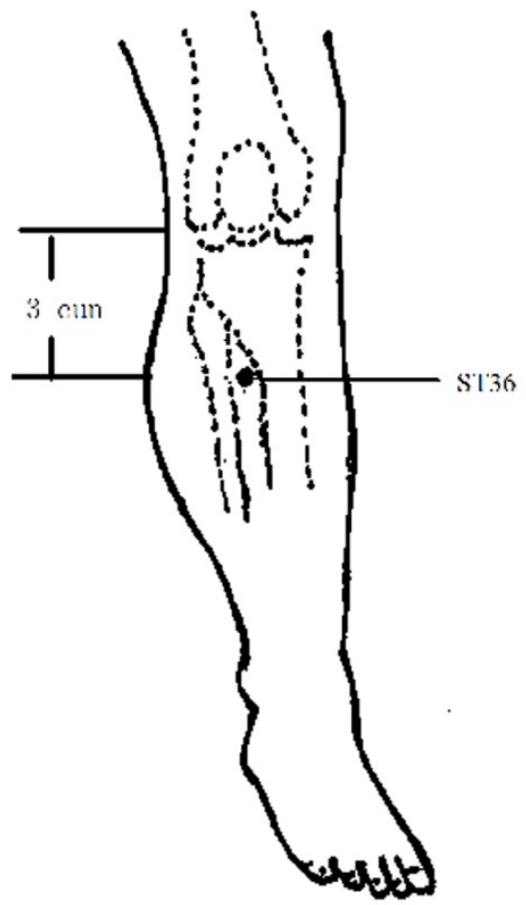

Good Manufacturing Practices, ISO 9002, EN46002 Quality certificate and CE certification for quality and safety. Needle size: $0.25 \times 30 \mathrm{~mm}$ needles in patients with normal BMI, $0.30 \times 40 \mathrm{~mm}$ needles in overweight patients. During acupuncture treatment, patients lay supine in their hospital bed. Skin was prepped with $70 \%$ alcohol. The skin was tightened by pressing around the target area, and the needle is then gently inserted. The needle was stimulated gently until "de qi" (a dull, aching, and spreading sensation). As soon as de qi is reached, the needle no longer causes pain or discomfort, at an insertion depth of about 1 to 2 cun. Needles were manually stimulated to evoke needle sensation upon insertion, at $10 \mathrm{~min}, 20 \mathrm{~min}$, and immediately prior to being removed at $30 \mathrm{~min}$. Acupuncture was continued once a day until recovery of peristalsis.

\section{ST36 acupoint injection}

This group received injection via syringe at the ST36 acupoint. Single-use, sterilized, $2 \mathrm{ml}$ volumetric syringes (Yuzhou, wuxi, China) conformed to the requirements of the United States FDA, and CE certification, for quality and safety. Patients lay supine position in their hospital bed, with bent knees. The ST36 acupuncture point was located and disinfected with $70 \%$ alcohol. When the needles are inserted into ST36 acupoint, patients feel de qi, stimulated to evoke needle sensation. Then $0.5 \mathrm{mg}$ neostigmine was slowly injected, injected at the other side using the same method. Acupoint injection treatment was continued once a day until recovery of peristalsis.

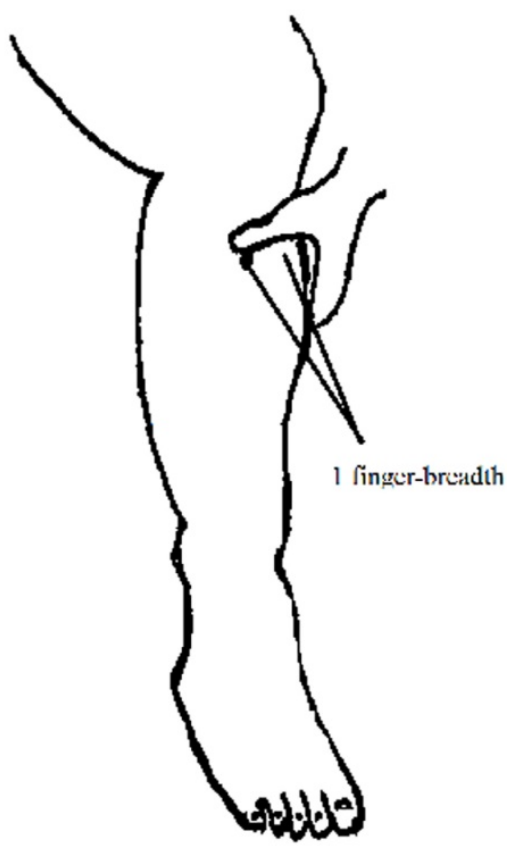

Figure 1. Location of ST36 


\section{Intramuscular injection}

Patients to lay in a lateral position in their hospital bed. Neostigmine $(1.0 \mathrm{mg})$ was injected into the gluteus maximus according to common practice. Intramuscular injection once daily, until recovery of peristalsis.

\section{Standard therapy}

Standard therapy included fasting, cessation of enteral nutrient solution, nasogastric suction therapy, intravenous fluids, parenteral nutrition, and gradual ambulation with simple exercises.

\section{Outcome measures}

Patients in the ST36 acupoint injection and intramuscular injection groups were auscultated for bowel sounds $10 \mathrm{~min}$ following injection. To avoid disturbing the therapy, patients in the ST36 acupuncture group were auscultated $30 \mathrm{~min}$ following initiation of acupuncture. Auscultation was performed in these three groups for 2 hours first, then all patients including the standard therapy group were auscultated once per hour. The four abdominal quadrants were auscultated for more than four minutes, and the number of bowel sounds were recorded. If the number of bowel sounds was more than three times per minute, peristalsis was considered recovered. Patients were asked to record the precise time that they first passed flatus and the precise time of their first bowel movement following surgery. Drug-related adverse events were assessed using a questionnaire. Adverse events included abdominal pain, diarrhea, nausea, vomiting, tearing, and anxiety.

The main outcome was effectiveness rate. Significant effectiveness was defined as first passed flatus or first defecation, and disappearance of abdominal distension within one hour of treatment. Effectiveness was defined as the same events occurring between one and two hours of treatment. If the patient did not pass flatus or have a bowel movement more than two hours following treatment, the intervention was considered ineffective. Total effectiveness rate was the rate of significant effectiveness with effectiveness. Treatments lasting longer than 96 hours without effect were considered failures.

Secondary outcomes were time to bowel sound recovery, time to first flatus, and time to first defecation. The latter outcomes were assessed by asking patients to record the precise date and time of first flatus and first defecation following surgery. Time 0 was defined as the time patients were diagnosed with PPOI and therapy was begun. We calculated the total number of hours between time 0 and the time of bowel sound recovery (more than three per minute), between time 0 and the passage of flatus, and between time 0 and the first bowel movement.

Tertiary outcomes were drug-related adverse events, assessed by questionnaire as above. Abdominal pain was assessed using a $0-10 \mathrm{~cm}$ visual analogue scale (VAS), with 0 representing no pain and 10 representing excruciating pain. Patients were asked to place a mark on the line at a point representing the severity of their pain using the anchors "no pain" and "pain as bad as it could be". Psychological conditions were evaluated using the Hospital Anxiety and Depression Scale (HADS). The 14-item HADS questionnaire evaluates severity of anxiety and depression using seven items for each affliction. Scores $\geq 8$ indicate probable anxiety or depression with great reliability and validity [24].

\section{Power calculation and sample size.}

To confirm the accurate size of the samples, we did a preliminary experiment. Base on the analysis of the preliminary experiment, we found that the significant effectiveness rate of the ST36 acupoint injection group was $90 \%(9 / 10)$, the intramuscular injection group was $60 \%(6 / 10)$. Generally, a level of significance of $\alpha=0.05$ and a power of $1-\beta=0.90$ were used, the sample size for this study was 57 in each arm. We recruited an additional 10 subjects to offset potential attrition.

\section{Statistical analysis}

Statistical analyses were performed with SPSS 16.0 for Windows (SPSS, Chicago, IL, United States). Continuous variables were expressed as mean $\pm \mathrm{SE}$, and categorical variables were expressed as percentage. Means were calculated using one way ANOVA; intergroup differences were analyzed using the Student's $t$ test for normally distributed continuous variables; intergroup differences in categorical data were assessed by the $x^{2}$ test. All statistical tests were two-sided and the level of significance was set at 0.05 .

\section{Results}

During the study period, 1046 patients underwent radical gastrectomy for primary tumor and D2 lymphadenectomy between September 2014 and March 2017 in the Department of Gastrointestinal Surgery of our institute. A total of 306 patients were diagnosed with PPOI. Of these, 38 were excluded according to our criteria. The remaining 268 participants were randomly assigned to receive ST36 acupuncture $(n=67)$, ST36 acupoint injection $(n=67)$, intramuscular injection $(n=67)$, or standard therapy 


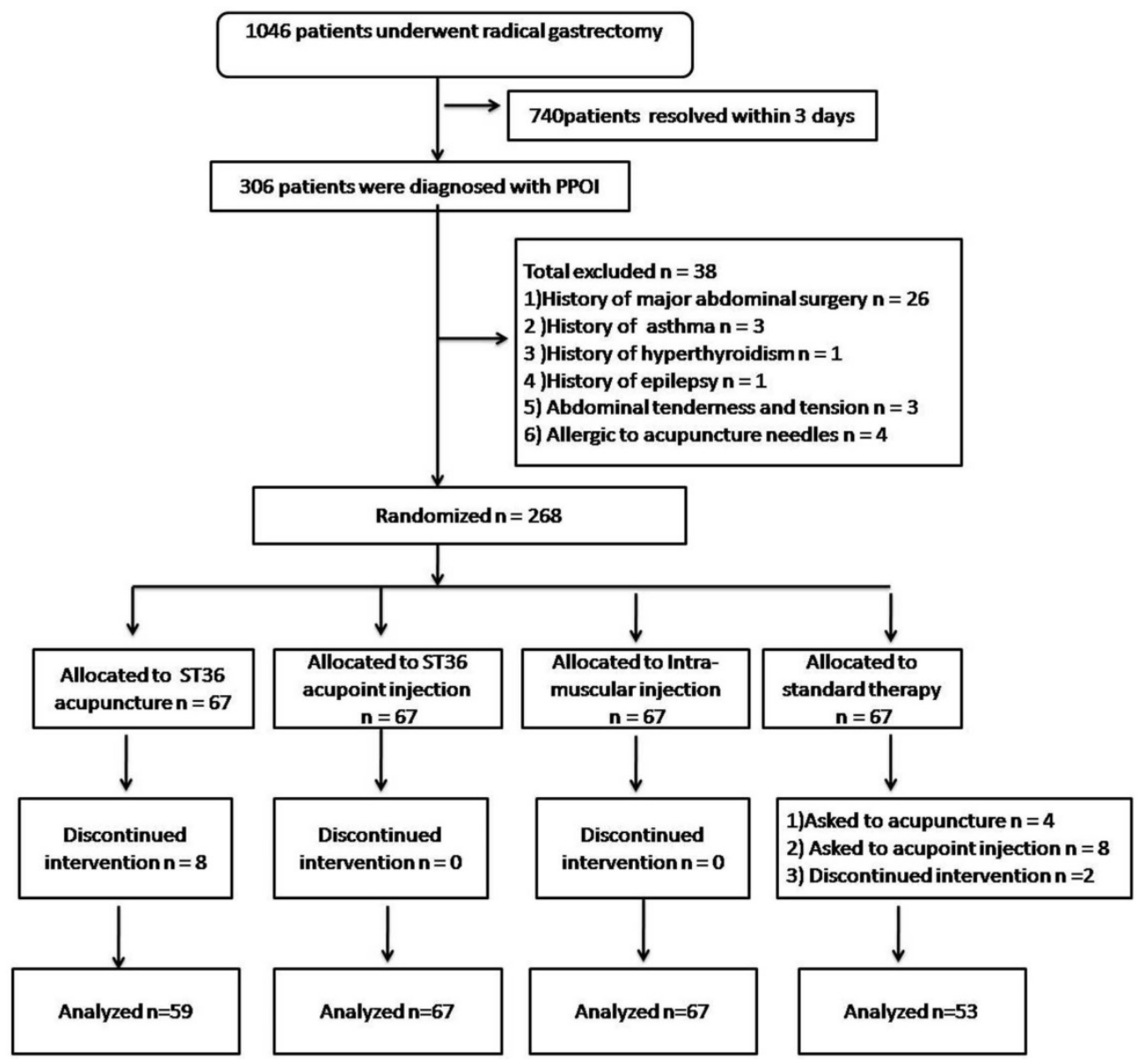

Figure 2. Flow diagram of study procedure for PPOI patients

$(n=67)$. Following randomization and therapy for more than 2 days, eight patients were unwilling to continue acupuncture. Fourteen were unwilling to receive standard therapy and asked for another method of therapy. The final analysis included 246 patients: 59 in the ST36 acupuncture group, 67 in the ST36 acupoint injection group, 67 in the intramuscular injection group, and 53 in standard therapy group (Figure 2).

Participant characteristics and baseline measures are shown in Table 1. Overall, there was no significant difference in the participant characteristics among all groups.

\section{Effectiveness rates}

In the ST36 acupuncture arm, one patient passed flatus within one hour following treatment, three patients passed flatus or defecated within two hours following treatment (total effectiveness rate $6.77 \%$ ). In the ST36 acupoint injection arm, 36 patients passed flatus or defecated within one hour following injection, 27 patients passed flatus or defecated within two hours following injection (total effectiveness rate $94.03 \%)$. In the intramuscular injection arm, 26 patients passed flatus or defecated within one hour following injection, 17 patients passed flatus or defecated within two hours following injection (total effectiveness rate $64.18 \%$ ). In the standard therapy arm, one patient passed flatus within one hour, and one patient defecated within two hours following treatment. The total effectiveness rate was $3.78 \%$. These results are displayed in Table 2. Compared to the standard therapy arm, the ST36 acupoint injection and intramuscular injection groups showed significantly higher effectiveness rates (all $P<0.01$ ), and the ST36 acupoint injection group showed significantly higher total effectiveness rate than that of the intramuscular injection group $(P<0.01)$.

\section{Time to bowel sound recovery, first flatus, and first defecation}

Compared to the group of standard therapy, other three groups showed shorter times to bowel sounds recovery, first flatus, and first defecation. However, only two groups of the ST36 acupoint injection and intramuscular injection showed significantly shorter times (all $P<0.01$ ). In which, the group of ST36 acupoint injection leaded to the best performance (all $P<0.01$ ). In the acupoint injection group, time to the group of bowel sound recovery is 
$1.88 \pm 0.51 \mathrm{~h}$, time to the group of first flatus is 2.30 $\pm 0.56 \mathrm{~h}$, and time to the group of first defection is 2.43 $\pm 0.61 \mathrm{~h}$, respectively. All groups of times to bowel sound recovery, first flatus, and first defecation are displayed in Table 3.

\section{Drug-related adverse events}

Drug-related adverse events are displayed in

Table 4. The ST36 acupoint injection arm showed significantly lower abdominal pain scores and a lower rate of diarrhea, nausea, vomiting, tearing, delirium, and anxiety than did the intramuscular injection arm $(P<0.05)$. No patient experienced seizures in either arm. Pain scores are shown in Fig. 3A. Anxiety and depression scores are shown in Fig 3B.

Table 1. Clinicopathological data of radical gastrectomy patients and diagnosed with PPOI followed by ST36 acupuncture, ST36 acupoint injection, intramuscular injection or standard therapy. * ASA, American Society of Anesthesiologists.

\begin{tabular}{|c|c|c|c|c|c|}
\hline Variable & Acupuncture $(\mathrm{n}=59)$ & Acupoint injection $(n=67)$ & Intramuscular injection $(n=67)$ & Standard therapy $(n=53)$ & $P$ value \\
\hline Age, yr & & & & & 0.380 \\
\hline$\geq 64$ & 33 & 37 & 41 & 24 & \\
\hline$<64$ & 26 & 30 & 26 & 29 & \\
\hline Gender & & & & & 0.664 \\
\hline Male & 38 & 50 & 46 & 37 & \\
\hline Female & 21 & 17 & 21 & 16 & \\
\hline Method of surgery & & & & & 0.839 \\
\hline Prox. gastrectomy & 3 & 9 & 7 & 5 & \\
\hline Distal gastrectomy & 35 & 35 & 37 & 28 & \\
\hline Total gastrectomy & 21 & 23 & 23 & 20 & \\
\hline Educational background & & & & & 0.235 \\
\hline None or primary school & 15 & 32 & 28 & 20 & \\
\hline Secondary school & 34 & 24 & 27 & 24 & \\
\hline University degree or above & 10 & 11 & 12 & 9 & \\
\hline Body mass index, $\mathrm{kg} / \mathrm{m}^{2}$ & & & & & 0.158 \\
\hline$<18.5$ & 3 & 15 & 9 & 6 & \\
\hline $18.5-25$ & 33 & 26 & 31 & 25 & \\
\hline$>25$ & 23 & 26 & 27 & 22 & \\
\hline ASA fitness grade* & & & & & 0.102 \\
\hline I & 31 & 53 & 42 & 36 & \\
\hline II & 27 & 13 & 24 & 16 & \\
\hline III & 1 & 1 & 1 & 1 & \\
\hline Type of surgery & & & & & 0.826 \\
\hline Laparoscopically assisted & 10 & 14 & 15 & 9 & \\
\hline Open & 49 & 53 & 52 & 44 & \\
\hline Surgical time, $\min$ & $180.76 \pm 12.68$ & $181.90 \pm 11.81$ & $182.55 \pm 9.90$ & $180.87 \pm 8.19$ & 0.767 \\
\hline Anemia state & & & & & 0.235 \\
\hline Without anemia & 5 & 12 & 3 & 4 & \\
\hline Mild anemia & 41 & 40 & 50 & 37 & \\
\hline Moderate anemia & 13 & 15 & 14 & 12 & \\
\hline Opioid analgesia use & & & & & 0.184 \\
\hline No & 7 & 12 & 18 & 12 & \\
\hline Yes & 52 & 55 & 49 & 41 & \\
\hline
\end{tabular}

Table 2. Effectiveness rate of ST36 acupuncture group, ST36 acupoint injection group, intramuscular injection group and standard therapy group.

\begin{tabular}{lllll}
\hline Variable & Acupuncture $(\mathrm{n}=59)$ & Acupoint injection $(\mathrm{n}=67)$ & Intramuscular injection $(\mathrm{n}=67)$ & Standard therapy $(\mathrm{n}=53)$ \\
\hline Significant effectiveness $(\%)$ & $1(1.69)$ & $36(53.73)$ & $26(38.81)$ & $1(1.89)$ \\
Effectiveness $(\%)$ & $3(5.08)$ & $27(40.30)$ & $17(25.37)$ & $0.939^{*},<0.01^{\dagger},<0.01^{\ddagger}, 0.083^{\#}$ \\
Total effectiveness (\%) & $4(6.77)$ & $63(94.03)$ & $43(64.18)$ & $0.363^{*},<0.01^{\dagger},<0.01^{\ddagger}, 0.066^{\#}$ \\
\hline
\end{tabular}

* Acupuncture VS. standard therapy. ${ }^{\dagger}$ Acupoint injection VS. standard therapy. ${ }^{*}$ Intramuscular injection VS. standard therapy. ${ }^{* A c u p o i n t}$ injection $V S$. intramuscular injection.

Table 3. The time to bowel sounds recovery, time to first flatus, and first defecation.

\begin{tabular}{lllll}
\hline Variable & Acupuncture $(\mathrm{n}=59)$ & Acupoint injection $(\mathrm{n}=67)$ & Intramuscular injection $(\mathrm{n}=67)$ & Standard therapy $(\mathrm{n}=53)$ \\
\hline T to bowel sound recovery, $\mathrm{h}$ & $35.54 \pm 2.67$ & $1.88 \pm 0.51$ & $7.15 \pm 1.21$ & $38.94 \pm 1.65$ \\
T to first flatus, $\mathrm{h}$ & $40.34 \pm 2.22$ & $2.30 \pm 0.56$ & $8.13 \pm 1.38$ & $0.281^{*},<0.01^{\dagger},<0.01^{\ddagger},<0.01^{\#}$ \\
T to first defecation, $\mathrm{h}$ & $47.44 \pm 1.56$ & $2.43 \pm 0.61$ & $9.78 \pm 1.66$ & $0.175^{*},<0.01^{\dagger},<0.01^{\ddagger},<0.01^{\#}$ \\
\hline
\end{tabular}

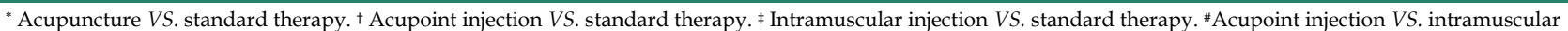
injection. 
Table 4. Drug-related adverse events. Intramuscular injection adverse events were more serious than ST36 acupoint injections $(P<0.05)$.

\begin{tabular}{llll}
\hline $\begin{array}{l}\text { Drug-related adverse } \\
\text { events }\end{array}$ & $\begin{array}{l}\text { ST36 acupoint } \\
\text { injection }(\mathrm{n}=67)\end{array}$ & $\begin{array}{l}\text { Intramuscular } \\
\text { injection }(\mathrm{n}=67)\end{array}$ & $P$ value \\
\hline Abdominal pain scores & $3.45 \pm 1.76$ & $5.27 \pm 1.86$ & $<0.01$ \\
Diarrhea(\%) & $27(40.30)$ & $38(56.72)$ & 0.042 \\
Nausea (\%) & $3(4.48)$ & $12(17.91)$ & 0.013 \\
Vomiting(\%) & $2(2.99)$ & $9(13.43)$ & 0.027 \\
Tearing(\%) & $1(1.49)$ & $7(10.45)$ & 0.031 \\
Delirium(\%) & $1(1.49)$ & $7(10.45)$ & 0.031 \\
Anxiety(\%) & $3(4.48)$ & $10(14.93)$ & 0.038 \\
\hline
\end{tabular}
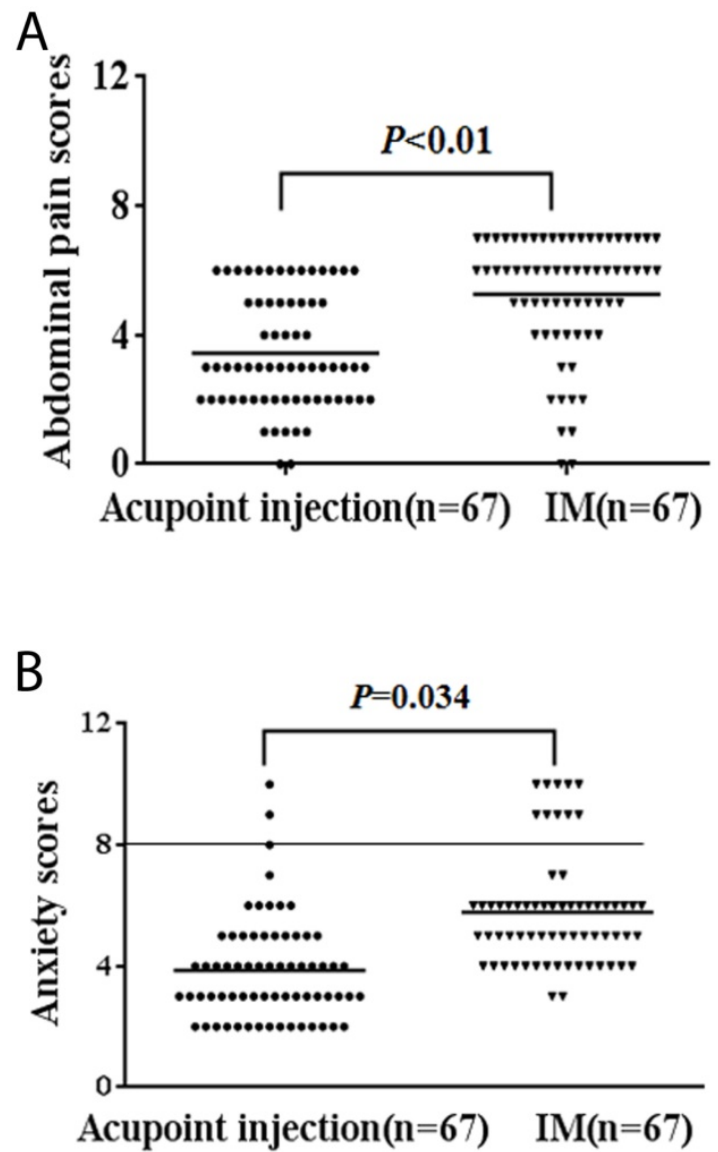

Figure 3. A, Abdominal pain scores of drug-related adverse events. B, Anxiety scores of drug-related adverse events. The intramuscular injection (IM) group gave more serious adverse than the ST36 acupoint injection group ( all $P<0.05$ ).

\section{Discussion}

In our study, PPOI developed in 306 patients, of whom 268 patients were eligible for enrollment. The incidence of PPOI following gastrectomy in our cohort is similar to numbers reported in previous studies [1].

The mechanism of POI is complex [25], characterized mainly by transient impairment of motility, dilation, and inflammatory infiltration [14]. In traditional Chinese medicine, acupuncture at the ST36 acupoint is thought to regulate the intestines. It has long been used to reduce the risk of POI and to manage various functional gastrointestinal disorders
[26-28]. A recent study showed that electroacupuncture reduced the duration of POI following laparoscopic surgery for colorectal cancer compared with control or sham acupuncture [29]. However, some studies found that acupuncture was not effective in prevention of POI. For example, Deng et al. conducted a randomized, sham-controlled trial to evaluate whether acupuncture reduces POI, and found that true acupuncture did not reduce POI more significantly than sham acupuncture [30]. Meng et al. found that acupuncture can be safely administered in a postoperative setting, but it was effective in preventing PPOI following intraperitoneal surgery for colon cancer [31].

To the best of our knowledge, there have been prior studies of acupuncture therapy in PPOI. Our study found that the effect of the treatment for PPOI only with do acupuncture is very limited, the total effectiveness rate of the acupuncture group was only $6.77 \%$, not significantly higher than the standard therapy group $\left(\chi^{2}=0.498, P=0.481\right)$. However, the total effectiveness rate of the ST36 acupoint injection with neostigmine group was significantly higher than that of the intramuscular acupuncture group $(P<$ 0.01). One explanation of this effect is that neostigmine is an acetylcholinesterase inhibitor, known to increase upper and lower gastrointestinal motility. When neostigmine is injected into the acupoint, it may amplify and sustain the effects of simple needling [32].

In order to further explore the mechanism of ST36 acupoint injection with neostigmine, we compared simple intramuscular injection with ST36 acupoint injection. In spite of the total effectiveness rate of intramuscular injection rising to $64.18 \%$, but it was significantly less than the effect of ST36 acupoint injection $(94.03 \%)(P<0.01)$. Agents administrated in acupoints, through the meridians, are thought to exert synergistic effects with acupoint stimulation, and are thought to give more sustained effects than traditional acupuncture needling or simple intramuscular injection [33].

We found the adverse events of ST36 acupoint injection group were significantly less than those of the intramuscular injection group $(P<0.05)$. According to one review [34], the strongest evidence for the use of ST36 acupuncture was for control of conditions such as anxiety, pain, nausea, and vomiting.

The present study has some limitations. One is that the patients were diagnosed clinically with PPOI, that is, without the benefit of objective diagnostic imaging. Likewise, treatment efficacy was not evaluated with imaging. A second limitation is the intra-abdominal pressure was not scientifically 
measured. In addition, there was no estimate of the change in intra-abdominal pressure prior to and following treatment. A third limitation is lack of complete blinding for patients and investigators. Such Nevertheless, nursing staff and statistician were blinded to treatment allocation throughout data collection and analysis. This limitation is shared with similar trials in other literature [35-37]. Blinding was judged impractical because of the nature of the interventions.

\section{Conclusion}

When neostigmine is injected into ST36 acupoint, it appears to amplify and sustain the effects of simple needling, significantly enhancing functional bowel recovery and reducing drug-related adverse events.

\section{Abbreviations}

GC: gastric cancer; HADS: Hospital Anxiety and Depression Scale; POI: postoperative ileus; PPOI: paralytic postoperative ileus; VAS: visual analogue scale.

\section{Acknowledgements}

This work was supported in part by the National Natural Science Foundation of China [grant numbers 81172279, 81572343].

\section{Competing Interests}

The authors have declared that no competing interest exists.

\section{References}

1. Huang DD, Zhuang CL, Wang SL, et al. Prediction of Prolonged Postoperative Ileus After Radical Gastrectomy for Gastric Cancer: A Scoring System Obtained From a Prospective Study. Medicine. 2015; 94: e2242. [PubMed:26705206]

2. Feldheiser A, Aziz O, Baldini G, et al. Enhanced Recovery After Surgery (ERAS) for gastrointestinal surgery, part 2: consensus statement for anaesthesia practice. Acta Anaesthesiol Scand. 2016; 60: 289-334. [PubMed:26514824]

3. Andersson T, Bjerså K, Falk K, et al. Effects of chewing gum against postoperative ileus after pancreaticoduodenectomy-a randomized controlled trial. BMC Res Notes. 2015; 8: 37. [PubMed: 25886536]

4. Vather R, Trivedi S, Bissett I. Defining postoperative ileus: results of a systematic review and global survey. J Gastrointest Surg.2013; 17: 962-72. [PubMed: 23377782]

5. Artinyan A, Nunoo-Mensah JW, Balasubramaniam S, et al. Prolonged postoperative ileus-definition, risk factors, and predictors after surgery. World J Surg. 2008; 32: 1495-500. [PubMed: 18305994]

6. Vilz TO, Pantelis D, Lingohr P, et al. SmartPill ${ }^{\circledR}$ as an objective parameter for determination of severity and duration of postoperative ileus: study protocol of a prospective, two-arm, open-label trial (the PIDuSA study). BMJ Open. 2016; 6: e011014. [PubMed: 27401360]

7. Holubar SD, Hedrick T, Gupta R, et al. American Society for Enhanced Recovery (ASER) and Perioperative Quality Initiative (POQI) joint consensus statement on prevention of postoperative infection within an enhanced recovery pathway for elective colorectal surgery. Perioper Med. 2017; 6: 4. [PubMed: 28270910]

8. Jung SY, Chae HD, Kang UR, et al. Effect of Acupuncture on Postoperative Ileus after Distal Gastrectomy for Gastric Cancer. J Gastric Cancer. 2017; 17: 11-20. [PubMed: 28337359]
9. Xue Q, Wang X-N, Deng J-Y, et al. Effects of extended lymphadenectomy and postoperative chemotherapy on node-negative gastric cancer. World J Gastroenterol. 2013; 19: 5551-6. [PubMed: 24023500]

10. Chan DC, Liu YC, Chen CJ, et al. Preventing prolonged post-operative ileus in gastric cancer patients undergoing gastrectomy and intra-peritoneal chemotherapy. World J Gastroenterol. 2005; 11: 4776-81. [PubMed: 16097043]

11. Oh CH, Ji GY, Yoon SH, et al. Paralytic Ileus and Prophylactic Gastrointestinal Motility Medication after Spinal Operation. Yonsei Med J. 2015; 56: 1627-31. [PubMed: 26446646]

12. You XM, Mo XS, Ma L, et al. Randomized Clinical Trial Comparing Efficacy of Simo Decoction and Acupuncture or Chewing Gum Alone on Postoperative Ileus in Patients With Hepatocellular Carcinoma After Hepatectomy. Medicine. 2015; 94: e1968. [PubMed:26559269]

13. Augestad KM, Delaney CP. Postoperative ileus: Impact of pharmacological treatment, laparoscopic surgery and enhanced recovery pathways. World J Gastroenterol. 2010; 16: 2067-74. [PubMed:20440846]

14. Endo M, Hori M, Ozaki H, et al. Daikenchuto, a traditional Japanese herbal medicine, ameliorates postoperative ileus by anti-inflammatory action through nicotinic acetylcholine receptors. J Gastroenterol. 2014; 49: 1026-39. [PubMed:23846546]

15. Han JS, Ho YS. Global trends and performances of acupuncture research. Neurosci Biobehav Rev 2011; 35: 680-7 [PubMed: 20800613].

16. [No authors listed]. World Health Organization: WHO Traditional Medicine Strategy 2002-2005. Geneva, Switzerland, World Health Organization, 2002.

17. Yin J, Chen JD. Gastrointestinal Motility Disorders and Acupuncture. Auton neurosci. 2010; 157: 31-7. [PubMed: 20363196]

18. Wang H, Wang L, Shi X, et al. Electroacupuncture at Zusanli Prevents Severe Scalds-Induced Gut Ischemia and Paralysis by Activating the Cholinergic Pathway. Evid Based Complement Alternat Med. 2015; 2015: 787393. [PubMed: 26448777]

19. Jung SY, Chae HD, Kang UR, et al. Effect of Acupuncture on Postoperative Ileus after Distal Gastrectomy for Gastric Cancer. J Gastric Cancer. 2017; 17: 11-20. [PubMed: 28337359]

20. Liu J, Huang $\mathrm{H}, \mathrm{Xu} \mathrm{X}$, et al. Effects and possible mechanisms of acupuncture at ST36 on upper and lower abdominal symptoms induced by rectal distension in healthy volunteers. Am J Physiol Regul Integr Comp Physiol 2012; 303: R209-17. [PubMed: 22592556]

21. Yong $\mathrm{C}$, Chen $\mathrm{S}$, Chen $\mathrm{H}$, et al. Central neuromechanisms underlying control of intragastric pressure through acupuncture at Zusanli (ST36) in rats: the upper cervical cord is the key link between the ascending and descending pathways. Neural Regen Res. 2016; 11: 971-6. [PubMed: 27482227 ]

22. MacPherson H, Asqhar A. Acupuncture needle sensations associated with De Qi: a classification based on experts ratings. J Altern Complement Med. 2006; 12: 633-7. [PubMed: 16970533]

23. Wang M, Gao YH, Xu J, et al. Zusanli (ST36) acupoint injection for preventing postoperative ileus: A systematic review and meta-analysis of randomized clinical trials. Complement Ther Med. 2015; 23: 469-83. [PubMed: 26051583]

24. $\mathrm{Li} \mathrm{XJ}, \mathrm{He} \mathrm{YL}, \mathrm{Ma} \mathrm{H}$, et al. Prevalence of depressive and anxiety disorders in Chinese gastroenterological outpatients. World J Gastroenterol. 2012; 18:2561-8. [PubMed: 22654455]

25. Ay AA, Kutun S, Ulucanlar H, et al. Risk factors for postoperative ileus. J Korean Surg Soc. 2011; 81: 242-9. [PubMed: 22111079]

26. Meng L, Chen S, Chen JD, et al. Effects of Transcutaneous Electrical Acustimulation on Refractory Gastroesophageal Reflux Disease. Evid Based Complement Alternat Med. 2016; 2016: 8246171. [PubMed: 27648103]

27. Zhang $\mathrm{F}$, Wu L, Zhao J, et al. Neurobiological Mechanism of Acupuncture for Relieving Visceral Pain of Gastrointestinal Origin. Gastroenterol Res Pract. 2017; 2017: 5687496. [PubMed: 28243252]

28. Zhou J, Fang $\mathrm{L}$, Wu WY, et al. The effect of acupuncture on chemotherapy-associated gastrointestinal symptoms in gastric cancer. Curr Oncol. 2017; 24: e1-e5. [PubMed: 28270726]

29. Melchart D, Streng A, Hoppe A, et al. Acupuncture in patients with tension-type headache: randomized controlled trial. BMJ. 2005; 331: 376-82. [PubMed: 16055451]

30. Deng G, Wong WD, Guillem J, et al. A Phase II, Randomized, Controlled Trial of Acupuncture for Reduction of Postcolectomy Ileus. Ann Surg Oncol. 2013; 20: 1164-9. [PubMed: 23188543]

31. Meng ZQ, Garcia MK, Chiang JS, et al. Electro-acupuncture to prevent prolonged ileus: a randomized clinical trial. World J Gastroenterol. 2010; 16: 104-11. [PubMed: 20039456]

32. Parthasarathy G, Ravi K, Camilleri M, et al. Effect of Neostigmine on Gastroduodenal Motility in Patients With Suspected Gastrointestinal Motility Disorders. Neurogastroenterol Motil. 2015; 27: 1736-46. [PubMed: 26387781] 
33. Zhu $\mathrm{YH}$, Chen $\mathrm{YH}$. On effects of acupoints and drugs in acupoint-injection treatment. Chin Acu Mox. 2005; 25: 46-8. [PubMed: 16309157]

34. Garcia MK, McQuade J, Haddad R, et al. Systematic Review of Acupuncture in Cancer Care: A Synthesis of the Evidence. J Clin Oncol. 2013; 31: 952-60. [PubMed: 23341529]

35. Song GM, Deng $\mathrm{YH}$, Jin $\mathrm{YH}$, et al. Meta-analysis comparing chewing gum versus standard postoperative care after colorectal resection. Oncotarget. 2016; 7: 70066-79. [PubMed: 27588405]

36. Ge B, Zhao H, Lin R, et al. Influence of gum-chewing on postoperative bowel activity after laparoscopic surgery for gastric cancer: A randomized controlled trial. Medicine. 2017; 96: e6501. [PubMed:28353600]

37. Atkinson C, Penfold CM, Ness AR, et al. Randomized clinical trial of postoperative chewing gum versus standard care after colorectal resection. Br J Surg. 2016; 103: 962-70. [PubMed: 27146793] 